\title{
Modelo de metodología para la enseñanza del baloncesto a los principiantes
}

\section{Methodology model for teaching basketball to beginners}

\section{INFORMACIÓN DEL}

\section{ARTÍCULO}

Fecha de recepción: 25 de Marzo de 2020.

Fecha de aceptación: 19 de Junio de 2020
1 Doctor en Ciencias Pedagógicas, Instituto de Cultura Física P. F. Lesgaft. Docente e Investigador de la Universidad de las Ciencias de la Cultura Física y el deporte "Manuel Fajardo"- Cuba.

E-mail:

ricardojosepardohernandez@gmail.com Código ORCID:

https://orcid.org/0000-0001-7141-9624

\section{Resumen}

Uno de los problemas de mayor incidencia en la enseñanza del baloncesto es que las metodologías que se utilizan no responden totalmente a las exigencias de la formación de los jugadores. Para dar solución a este problema, se llevó a cabo esta investigación con el objeto de elaborar un modelo de metodología del proceso de enseñanza del juego con un enfoque integral. En tal sentido, se emplearon los siguientes métodos: analítico - sintético, inductivo deductivo y modelación, que permitieron organizar el proceso investigativo. Como resultado se presenta un modelo de metodología denominado Padbal; elaborado sobre la base de fundamentos didácticos y orientado a desarrollar el proceso de aprendizaje en dos fases, con objetivos, contenidos y características propias, lo que facilita la programación de la enseñanza del baloncesto, tanto en el proceso de entrenamiento de jugadores principiantes, como en la educación física.

\section{Palabras Clave:}

Baloncesto, enseñanza, metodología, principiante, proceso, modelo.

Clasificación JEL: I2.

\begin{abstract}
One of the problems identified with the greatest incidence in basketball teaching today is that the methodologies used do not fully respond to the demands of the training of the players. To solve this problem, an investigation was carried out with the objective of developing a methodology model with a comprehensive approach to the game teaching process. In this sense, the following methods were used: analytical synthetic, inductive - deductive and modelling, which allowed organizing the investigative process. As a result of the investigation, a methodology model called Padbal is presented; elaborated on the basis of didactic foundations and oriented to develop the learning process in two phases. With its own objectives, contents and characteristics, which facilitates the programming of basketball teaching, both in the training process for beginning players, and in physical education.
\end{abstract}

Keywords:

Basketball, beginner, methodology, process, teaching, model.

JEL Classification: I2.

\footnotetext{
PODIUM No. 37, Junio 2020, pp. 107-128

(C) Universidad Espíritu Santo - UEES

ISSN: 1390-5473 e-ISSN: 2588-0969
} 


\section{Introducción}

Existe un marcado interés en la comunidad de especialistas, atletas, dirigentes y amantes del baloncesto, por alcanzar a nivel internacional resultados competitivos superiores; para lo que se requiere del desarrollo de métodos modernos de enseñanza de este deporte que permitan maximizar el potencial natural de atletas talentosos y lograr el éxito. El baloncesto, como parte de la educación física y el deporte contemporáneo lleva consigo nuevas exigencias en el proceso de su enseñanza. Para el desarrollo de dicho proceso, los especialistas utilizan fundamentalmente dos metodologías basadas en los modelos de enseñanza: tradicional o de instrucción directa y constructivista o de indagación (Gamero, 2015; Peráček y Peráčková, 2018), las cuales no dan respuesta totalmente a los requerimientos de la enseñanza de este deporte.

Por tales motivos, justifica la realización de la presente investigación teórica la necesidad de contar con un instrumento pedagógico que posibilite desarrollar el proceso de enseñanza del juego; de forma tal, que satisfaga en la dimensión necesaria, la formación en el jugador de la orientación fundamental de la acción (OFA), categoría subjetiva que existe en la conciencia o en el subconsciente del sujeto como reflejo de las condiciones en que se produce su acción en el juego, la cual determina en lo fundamental, el éxito en su ejecución (Bogen, 1985; Famose, 2015).

La citada situación problemática, motivó plantear el siguiente problema científico: ¿Cuáles serán los criterios a considerar en la concepción de un modelo de metodología para la enseñanza del baloncesto a los principiantes, que facilite en los jugadores la formación de la orientación fundamental de la acción en el juego? Para darle respuesta se planteó como objetivo de la investigación: Elaborar un modelo de metodología para la enseñanza del baloncesto a principiantes, que propicie la formación de la orientación fundamental de la acción en los jugadores. Con tal fin, se establecieron las siguientes preguntas científicas: 1) ¿Qué características presentan las metodologías para la enseñanza del baloncesto en los últimos tiempos?, 2) ¿Cuáles son los criterios teóricos, metodológicos y prácticos a considerar en la concepción de un modelo de metodología para la enseñanza del baloncesto, que facilite en el jugador la formación de la orientación fundamental de la acción en el juego?, y 3 ) ¿Cómo operar con los criterios teóricos, metodológicos y prácticos para concebir un modelo de metodología integral para la enseñanza del baloncesto?

\section{Revisión de literatura}

La metodología de la enseñanza del baloncesto se ha venido abordando en el de- cursar del tiempo, mediante dos modelos de enseñanza deportiva y en estrecha relación con los cambios del reglamento y los avances técnicos y tácticos del juego. Uno de los modelos de enseñanza que los profesores utilizan en su trabajo, es el modelo tradicional que se caracteriza en su aplicación por transitar: 
de lo técnico a lo táctico, de situaciones aisladas o modificadas a situaciones de juego real o contextualizadas, de lo individual-analítico a lo colectivo-global, del cómo al porqué y para qué del juego y de lo particular a lo general. En la práctica, este enfoque no ha facilitado la aplicación del conocimiento técnico en la acción táctica, reteniendo la capacidad de los jugadores principiantes de solucionar situaciones de juego, en muchos casos, debido al desconocimiento inicial del para qué de la acción que se aprende. Además, generalmente los contenidos que se tratan no abarcan con la profundidad necesaria, importantes aspectos como son: la enseñanza de la técnica como preparación para la enseñanza de la táctica, la apropiación de conceptos de juego, la educación de valores en los entrenamientos y el juego, y el desarrollo de la preparación física requerida para la actividad.

El modelo constructivista, también llamado integrado y sus corrientes, utilizado en la práctica pedagógica, resulta ser una alternativa al modelo tradicional de enseñanza deportiva. Este propone en el inicio del proceso de enseñanza: la presentación del juego, el desarrollo de la motricidad, y el descubrimiento del fundamento técnico a través del juego; donde surgen problemas tácticos que implican el análisis, reflexión y toma de decisiones en la resolución del problema planteado. Después se pasa a la etapa de racionalización, la cual tiene como objetivo conocer y dominar el fundamento hasta el nivel de hábito motor y el dominio de las acciones tácticas (Alonso, 2016; Gamero, 2015; Ibáñez, Feu, y García-Rubio, 2019). En el citado proceso, el alumno debe realizar una cantidad de pruebas, para encontrar la solución correcta. En este caso, la aplicación del método ensayo-error puede detener la enseñanza por un largo tiempo; incluso a veces la tarea de estudio no se logra resolver. Además, en su puesta en práctica, se observa con frecuencia la imposibilidad de los principiantes de ejecutar de forma adecuada los elementos técnicos y tácticos, debido a que no poseen un conocimiento anterior que sirva de base para la realización de los mismos, poniéndose de manifiesto la subvaloración del papel del hábito motor en la realización de acciones motrices.

Las investigaciones acerca de la enseñanza de este juego muestran la importancia del problema en el contexto científico. En la búsqueda de nuevas estrategias para la iniciación de niños y adolescentes en el juego, las investigaciones de Salazar (2013) y Moti y Wolde señalan (2018) exponen en sus resultados, la necesidad de contar con modelos alternativos que faciliten la programación del proceso de enseñanza-aprendizaje; así como la urgencia en bridar capacitación metodológica a los encargados de dirigir las categorías del minibaloncesto en los territorios estudiados.

Gran interés despierta el estudio sobre el proceso de enseñanza-aprendizaje del baloncesto en las escuelas deportivas, en el cual Víllora, Gutiérrez, Viñedo, y Bustos (2007) afirman que la elaboración 
y organización del proceso de enseñanza-aprendizaje debe llevarse a cabo estructurando etapas de desarrollo, es decir concretando qué objetivos, contenidos, instrumentos de evaluación o tipo de competición que se va a desarrollar a lo largo de un año o una temporada deportiva, lo que debe considerarse un importante aspecto en la elaboración de una metodología superior a las existentes. Se hace difícil sobreestimar el valor de los resultados de la investigación de los autores González, Ibáñez, y Feu (2017), donde se analizan dos programas de intervención, uno basado en la Instrucción Directa y otro en los Tactical Game Approach (TGB) o enseñanza basada en la táctica, con enfoque similar a la metodología constructivista, arribando a la conclusión de que ambos programas son válidos $\mathrm{y}$ fiables, teniendo el programa TGB mayores beneficios en aspectos técnicos, tácticos, físicos $\mathrm{y}$ afectivos que el programa DIB. En estos resultados se pone en evidencia el valor de la enseñanza táctica en el proceso de formación de jugadores, comprobado en otros estudios (Fessia, 2016), y justificado por la incapacidad de los noveles jugadores de aplicar en situación real de juego los aprendizajes solo a través de ejercicios de técnica individual sin complejidad ni oposición. Otros estudios abordan problemas particulares de la enseñanza del deporte de las canastas y las cestas como, por ejemplo, el referido al estudio de la efectividad de la aplicación en niños de quinto y sexto grado de una metodología de entrenamiento que incluye varios ejercicios para el desarrollo de cualidades de velocidad-fuerza; así como ejercicios para el desarrollo de diferentes cualidades físicas al mismo tiempo, obteniéndose resultados satisfactorios. Lo que corrobora la relación existente entre las capacidades físicas y la aplicación de las habilidades especificas en el juego (Popclevich, 2019).

Partiendo del criterio de que la enseñanza tradicional del baloncesto presenta problemas, como por ejemplos: la duplicación de contenidos, métodos obsoletos, la desconexión entre teorías y prácticas, y la falta de aplicación de la tecnología informática y de Internet, se puso en práctica en la Universidad Normal de Shandong en la provincia de Shandong China, el modo de enseñanza de baloncesto universitario LAMS. El cual incluye principalmente actividades programadas y utilización de herramientas de aprendizaje; demostrándose que puede mejorar significativamente el efecto de la enseñanza de baloncesto universitario (Cheng y Sheng, 2018). Dado el nivel de enseñanza donde se aplica este sistema, no es factible su aplicación, por el momento, en el nivel escolar al cual se dirige este trabajo; no obstante, el uso de recursos informáticos debe tenerse en cuenta en el proceso que se estudia.

Dando respuesta a la pregunta científica de ¿qué características presentan las metodologías para la enseñanza del baloncesto en los últimos tiempos? Se puede resumir que las investigaciones citadas muestran la problemática existente en cuanto a las carencias metodológicas de los modelos 
de enseñanza del baloncesto que generalmente se utilizan a nivel de la iniciación deportiva; no obstante, no se presenta un enfoque alternativo de programación del proceso en cuestión, que dé respuesta a las exigencias actuales de la formación del baloncestista principiante.

Documentos y estudios acerca de la enseñanza del baloncesto en el nivel de iniciación deportiva

Uno de los trabajos que brinda una valiosa información sobre el desarrollo de la enseñanza del juego, es el Manual para los Institutos de Cultura Física de la URSS donde se expone, en cuanto al período del establecimiento inicial de la técnica del juego, un ordenamiento del proceso de enseñanza que abarca desde la utilización de movimientos naturales, hasta la aplicación en el juego de los fundamentos técnicos; así como, ejercicios para la enseñanza de cada elemento técnico por separado $\mathrm{y}$ combinado. De igual forma, aborda la preparación táctica, sus tareas en la etapa de dominio inicial de la táctica del juego y un ordenamiento en la enseñanza de las acciones individuales, de grupo y equipo ofensivas y defensivas; además trata diferentes formas de desarrollar la preparación de juego y competitiva. Sin embargo, deja de considerar en la programación de la enseñanza, el potencial inicial de aprendizaje de los contenidos de los principiantes, así como; la educación de los conceptos de juego, las relaciones entre objetivos - contenido - método - estrategias y evaluación, lo que no garantiza la debida información acerca de progresión del aprendizaje en cuestión (Cemachko,1976). En diferentes trabajos se encontró información acerca de la técnica de ejecución de acciones del juego y la metodología para la enseñanza de los fundamentos técnicos y acciones tácticas (Basketball for Coaches, 2020; Erculj y Strumbelj, 2015; Mondoni, 2017). Llama la atención el folleto del curso Enseñanza del Baloncesto impartido por la Cárdenas et al. (2015), en el cual se brinda una interesante información sobre aspectos del proceso de enseñanza y entrenamiento de este deporte en principiantes, como por ejemplo: la formación motriz básica, enseñanza significativa, aprendizaje funcional, motivación, papel del profesor como mediador del aprendizaje, estos ayudan a comprender las características que debe tener el trabajo del entrenador en este nivel. El proceso de planificación del entrenamiento también se aborda, el tema referente a la evaluación de la enseñanza aporta procedimientos a tener en cuenta, en cuanto a la evaluación sistemática del juego y los tipos de observaciones, aquí se recomienda considerar los siguientes aspectos: situación y conductas a observar de los jugadores, medios para su registro y análisis de los resultados.

El programa de baloncesto para grupos de iniciación en este deporte en Rusia, incluye: el contenido teórico y práctico, su distribución anual y los criterios de evaluación (Irina, 2015), elementos de marcada utilidad en el proceso que se estudia.

A pesar del alto valor que en el orden 
metodológico tiene la bibliografía mencionada, esta no satisface totalmente la necesidad de una orientación pedagógica específica para llevar a cabo el proceso de enseñanza del baloncesto con principiantes, ya que generalmente se hace referencia a los contenidos a impartir y no a la marcha y dinámica del citado proceso, pero sí constituyen estos trabajos una fuente de elementos a considerar, para la elaboración de un modelo de metodología que de forma integral aborde el aprendizaje del juego. En la construcción de un modelo de metodología para la enseñanza del baloncesto, es imprescindible tener en cuenta los principios didácticos generales aplicados a la enseñanza de las acciones motoras; ellos constituyen una interpretación y aplicación en la educación física y el deporte de los principios didácticos generales de la pedagogía, al reflejar las leyes de la didáctica que determinan la marcha de la enseñanza en su totalidad y sus partes (Pavlovich, 2019).

El análisis de la bibliografía referente al proceso de enseñanza aprendizaje de las acciones motrices, permite comprender con mayor profundidad las características que debe observar la formación de habilidades deportivas del baloncesto y los procesos cognitivos que permiten al baloncestista la toma de decisiones en el juego.

Diferentes autores a través del tiempo han abordado la enseñanza de las acciones motrices. Las ideas de Sechenov, en Rusia en el siglo XIX sobre el mecanismo de la enseñanza desde la posición de la fisiología, sirvieron como núcleo para los trabajos de Pavlov (1800-1900) y sus discípulos acerca de la investigación de los mecanismos de la formación de los reflejos condicionados. Torndike planteó los principios de la concepción del biorritmo en la enseñanza (prueba, error, y éxito casual como camino principal de la enseñanza). Anojín al desarrollar la teoría de Pavlov fundamentó la concepción de la acción aferente y eferente, lo que permitió la posibilidad de prever el resultado de la acción y corregirla en el transcurso de su ejecución. De 1930 a 1940, Bernchteín elabora un estudio sobre la construcción del movimiento que posteriormente queda como base de la fisiología activa. En 1930 Samoilov formula la concepción del reflejo aislado, lo que explica la coordinación del movimiento; el confirmó que el movimiento voluntario se dirige no sólo por el carácter reflejo, sino también por la conciencia. Entre 1950 y 1960, Galpierin elaboró la teoría de la formación de las acciones y los conceptos por etapas; donde cada acción está compuesta de tres etapas: orientación, realización y controlcorrección (Bogen,1985).

Gran importancia reviste las posiciones de Bogen (1985) acerca del proceso de aprendizaje de la acción motriz, cuando explica que el profesor no solo debe prestar atención a la realización de las particularidades de la acción y sus puntos fundamentales de apoyo (PFA), o partes más importantes de la acción, sino también, a las condiciones en que se desarrolla la tarea motora, de forma tal que el jugador aprenda a leer el juego y en 
dependencia de esto actuar, es decir formar la orientación fundamental de la acción (OFA). En la enseñanza mal organizada, cuando la formación de la OFA sucede fuera de la dirección pedagógica, el proceso se retarda por largo tiempo, a veces años. Si el profesor dirige la formación de la OFA, entonces el plazo de su formación se acorta hasta en diez veces y se reduce el plazo de la enseñanza con una calidad superior. $\mathrm{Al}$ desarrollarse el proceso de enseñanza del juego en el marco del entrenamiento deportivo de niños y jóvenes, las principales direcciones de la preparación deportiva (Platonov, 2014) también condicionan la programación del aprendizaje.

Un enfoque que permite entender las particularidades de la enseñanza, lo brinda Pardo (2007) quien señala que las tareas generales de la enseñanza del baloncesto que deben cumplir los alumnos en las canchas bajo la guía del profesor, en la etapa de establecimiento inicial de las bases para poder jugar, son: a) Conocimiento de las generalidades del juego. b) Educación de la motricidad general, c) Dominio de la técnica fundamental del juego. Realización de acciones técnicas combinadas, en situaciones complejas, con oposición y similares al juego, d) Dominio de las acciones tácticas elementales de tipo individual, de grupo y equipo, e) Educación de valores, conceptos de juego, conocimientos sobre el reglamento y otros importantes aspectos teóricos, f) Desempeño adecuado en un juego oficial o con reglas especiales, g) Desarrollo de la preparación física requerida para la actividad, y $\mathrm{h}$ ) Evaluación de la enseñanza y el juego.

Al elaborarse un modelo integral de metodología (Padbal), como instrumento pedagógico que posibilite la programación del proceso de enseñanza del deporte de las cestas y las canastas, acorde con sus exigencias, permitirá a los profesores y entrenadores dirigir con mayor efectividad la enseñanza de este deporte en las escuelas e instituciones deportivas, teniendo como impacto, el incremento de practicantes y la mejor formación de los talentos deportivos.

\section{Metodología}

La presente investigación está orientada a dejar una base teórica para que futuros investigadores hagan una aplicación práctica de lo indagado. El período de desarrollo del trabajo de investigación se enmarcó, entre el mes de julio del año 2018 y febrero del 2020 . Con el fin de dar respuesta a las preguntas científicas de la investigación, se utilizaron en el trabajo los métodos siguientes (Styopin, Yelsukov, y Goldberg, 2020):

\section{a) Analítico - sintético}

Este método permitió valorar los aspectos positivos $y$ negativos que presentan los enfoques metodológicos para la conducción del proceso de enseñanza del baloncesto, y a la vez determinar los componentes a tener en cuenta en la construcción de un modelo de metodología de enseñanza del juego. Con tal fin, se utilizó como 
procedimiento el análisis crítico de la bibliografía especializada en el tema, para establecer juicios y valoraciones acerca de las categorías esenciales que participan como fundamentos de la metodología que se propone. Los instrumentos utilizados en la investigación con el propósito de recolectar la información necesaria fueron los siguientes: ficha bibliográfica, ficha hemerográfica, y registro de páginas electrónicas (Castillo,2019).

\section{b) Inductivo - deductivo}

Este método se aplicó principalmente, en el razonamiento y comprensión de las relaciones entre los fundamentos del proceso de enseñanza del juego y sus particularidades.

\section{c) Modelación}

Para llevar a cabo la representación de este complejo proceso de enseñanza aprendizaje se acudió a la modelación como método científico, la que posibilitó determinar los presupuestos teóricos de partida en la construcción del modelo de la metodología propuesta; así como, reproducir y analizar las relaciones de los elementos que están inmersos en el proceso en cuestión.

Las variables de la investigación fueron determinadas teniendo en cuenta el problema científico declarado: ¿Cuáles serán los criterios a considerar en la concepción de un modelo de metodología para la enseñanza del baloncesto a los principiantes, que facilite en los jugadores la formación de la orientación fundamental de la acción en el juego? Así como la naturaleza del objeto a investigar: el proceso de enseñanza aprendizaje del baloncesto; teniendo presente que la variable es una representación de la realidad creada por el investigador y la aporta el investigador de acuerdo con sus necesidades, las cuales incluyen no sólo la realidad objeto de investigación, sino también la intención que persigue el estudio reflejada en los objetivos (Carballo y Guelmes, 2016). Las variables de la investigación, son las siguientes:

a) Fundamentos didácticos del proceso de enseñanza del baloncesto para principiantes.

Entiéndase, como las teorías que estudian la organización del proceso de enseñanza del baloncesto en principiantes.

b) Contenido general del proceso de enseñanza del baloncesto para principiantes.

El contenido general del proceso de enseñanza del baloncesto para principiantes se comprende, como los saberes teóricos y prácticos, así como las experiencias relacionadas con la práctica del baloncesto por principiantes.

c) Estrategia didáctica del proceso de enseñanza aprendizaje del baloncesto en principiantes.

Se define a la estrategia didáctica del proceso de enseñanza aprendizaje del baloncesto en principiantes, como el 
sistema de acciones y operaciones seleccionadas y organizadas en forma de tareas docentes, que le permitan al deportista apropiarse de los métodos de solución de problemas que se presentan en la práctica del baloncesto en el nivel de iniciación deportiva.

d) Métodos para la enseñanza del baloncesto.

Los métodos para la enseñanza del baloncesto son los modos que se vale el docente, para dirigir la actividad consciente del deportista en el proceso de enseñanza-aprendizaje de este deporte.

e) Relaciones entre los objetivos contenidos a impartir - estrategias de aprendizaje - métodos - medios procedimientos y evaluación de la enseñanza y del juego.

Las relaciones entre los objetivos contenidos a impartir - estrategias de aprendizaje - métodos - medios procedimientos y evaluación de la enseñanza y del juego, se definen como los vínculos que se establecen entre los componentes del subsistema didáctico de la enseñanza del baloncesto a principiantes.

Los pasos seguidos para llegar a los resultados esperados fueron:

a) Elaboración del diseño preliminar de la investigación.

b) Estudio de las variables de la investigación, evaluando los aspectos positivos y negativos que estas presentan en los actuales enfoques metodológicos para la conducción del proceso de enseñanza del baloncesto en principiantes.

c) Determinación de los componentes a tener en cuenta en la construcción de una propuesta de modelo de metodología integral de enseñanza del juego (fundamentos didácticos, contenidos generales, fases del proceso de enseñanza, métodos $\mathrm{y}$ orientaciones metodológicas).

d) Modelación de la metodología de enseñanza del juego (componentes de la metodología y sus relaciones), en correspondencia con las características de la enseñanza motivo de estudio.

\section{Resultados}

Como resultado de los pasos seguidos en la investigación, se determinaron los criterios a considerar en la concepción de un modelo de metodología integral para la enseñanza del baloncesto en los principiantes:

1. Los fundamentos didácticos que deben observarse en el proceso de enseñanza del baloncesto para principiantes.

Son los siguientes: a) Principios didácticos generales aplicados a la enseñanza de las acciones motoras (Pavlovich, 2019), b) Principios generales y especiales de la preparación deportiva (Platonov, 2014), c) Teoría de Galperin sobre la dirección del aprendizaje de los conocimientos, la formación de las 
acciones y los conceptos (Bogen, 1985), d) Tareas generales de la enseñanza del baloncesto (Pardo, 2007), y e) Evaluación del proceso de enseñanza y del juego (Vladimirovich, 2015).

La esencia de este modelo de metodología, radica en la aplicación práctica de sus fundamentos en cada una de las fases que se establecen para el proceso de enseñanza - aprendizaje del juego, las cuales poseen tareas, métodos a utilizar y estrategias didácticas propias (ver Figura 1).

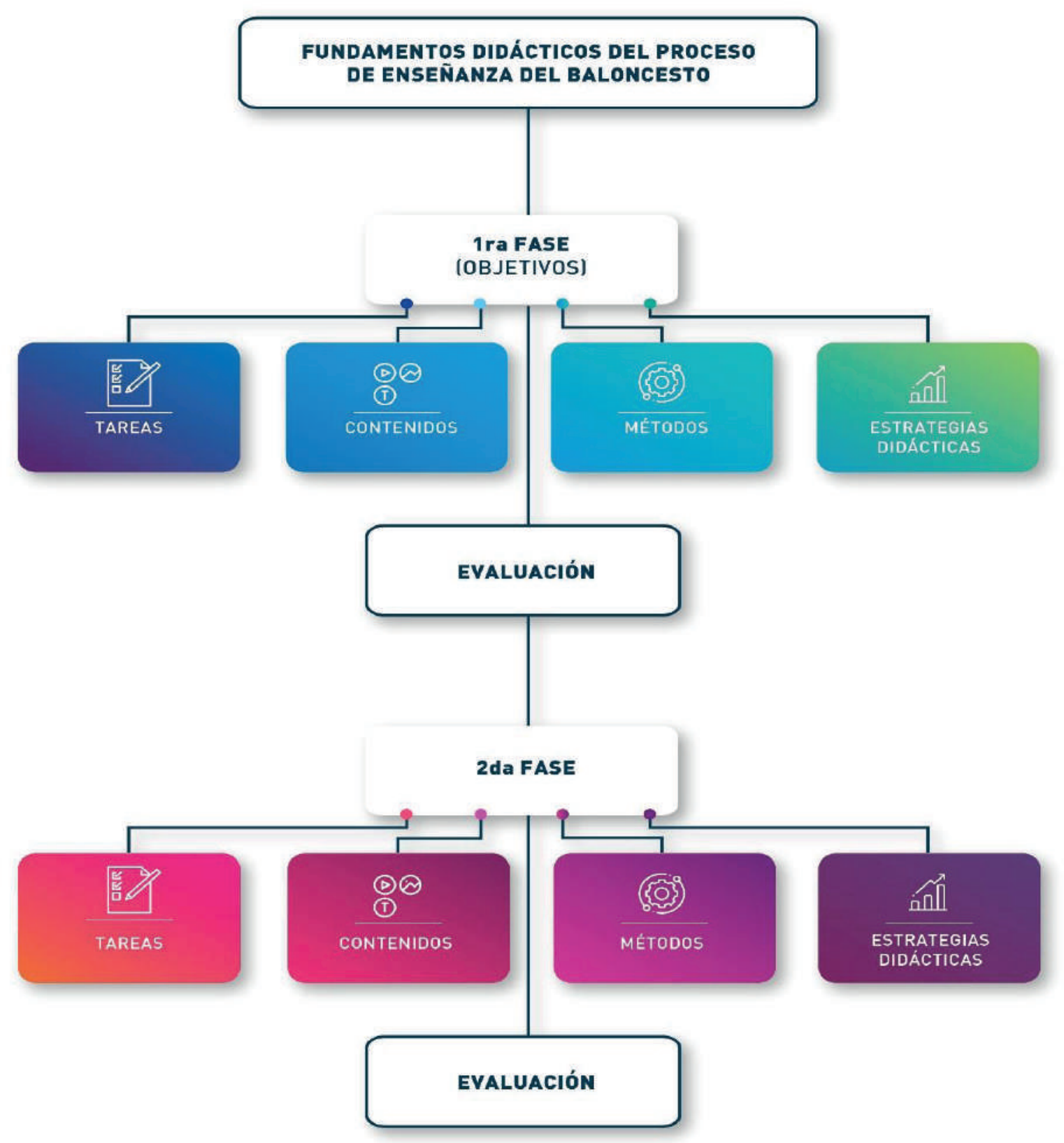

Figura 1. Modelo de metodología para la enseñanza del baloncesto. Fuente: Elaboración propia. 
2. Contenido general del proceso de enseñanza del baloncesto para principiantes.

Partiendo de que la actividad pedagógica en la enseñanza del baloncesto debe estar dirigida, en primer lugar, a la mejora interconectada de los componentes significativos de la actividad del juego, el contenido general a impartir en las etapas de la propuesta, es el siguiente:

- Presentación del juego: Donde se abordan temas relativos a: esencia del juego, reglas fundamentales, y otros elementos de la actividad, utilizando para esto los métodos orales y demostrativos.

- Motricidad general y aplicada al baloncesto: La cual tiene como propósito, construir los cimientos necesarios en el plano motor, para desarrollar las habilidades relacionadas específicamente con este deporte (Gayubo, 2017).

- Estudio de los fundamentos técnicos por separado: Esto se presenta al comienzo y en cada clase del proceso, para lo cual se utilizan principalmente los siguientes métodos: explicación, demostración, repetición, competitivo y juego, con la aplicación de gran número de ejercicios prácticos (Pardo, 2007).

- Fundamentos técnicos de forma combinada: Después que el alumno aprende a realizar dos o más fundamentos, se inicia su estudio de forma combinada.

- Estudio de la técnica como preparación para la táctica: Comprende la ejecución de elementos y combinaciones técnicas en situaciones complejas, como preparación para la enseñanza de la táctica.

- Dominio de las acciones tácticas elementales ofensivas y defensivas: $\mathrm{El}$ estudio de las acciones tácticas individuales se lleva a cabo posterior al estudio de los fundamentos técnicos que le corresponden. Para el estudio de las acciones tácticas individuales se sugieren diferentes métodos (oral, demostrativo, problémico, de acciones simplificadas de juego, repetición).

En cuanto al estudio de las acciones tácticas de grupo, se presenta un ordenamiento de estas en la ofensiva y defensiva, de la siguiente forma: a) Primero se forman los hábitos de las acciones de la ofensiva de posición. b) Al mismo tiempo, se estudia la ofensiva rápida. c) Después se tratan la defensa personal y de presión personal. d) A continuación, se aborda la defensa de zona y sus variantes (Cemachko,1976).

- Juego oficial y de estudio: Desde las primeras clases, se realizan juegos aplicando las reglas y los fundamentos técnicos y tácticos aprendidos.

- Preparación física requerida: La ejecución de las acciones de juego eleva el nivel de las capacidades físicas del practicante y a la vez requiere, para su mejor ejecución, del desarrollo de los niveles del potencial motor del jugador.

El entrenamiento en habilidades de 
baloncesto se considera exitoso sólo si los estudiantes pueden usar efectivamente su potencial motor en un juego de confrontación. La formación de interconexiones entre los componentes individuales de la preparación es la base para el entrenamiento de las actividades de juego. La tarea del maestro es enseñar a sus alumnos en el juego a maximizar sus habilidades físicas al realizar técnicas.

- Educación en valores: En la dirección del proceso está presente la labor del profesor que comprende la educación de valores, principios y convicciones.

- Preparación teórica: La transmisión a los alumnos de conocimientos específicos del baloncesto y de la cultura física en general, entre otros temas, comprende este importante aspecto de los contenidos de la enseñanza. En el desarrollo de esta preparación se recomienda el estudio de las reglas más importantes del juego, ya que su conocimiento determina, en gran medida, las habilidades técnicas y tácticas necesarias para poder jugar (Rebollo, Vizcaíno, Sáenz-López, y Fernández, 2015). El uso de recursos informáticos (videos, multimedia y software, entre otros) puede contribuir en el desarrollo de la preparación teórica de los jugadores (Cheng and Sheng, 2018).

- Evaluación de la enseñanza y el juego: La evaluación del aprendizaje y el juego, tiene como propósito conocer, qué ayuda necesitan los alumnos para alcanzar los logros que de ellos se espera en cada fase. La información que el profesor obtendrá mediante la aplicación de los instrumentos evaluativos de registro de la conducta de los jugadores en los ejercicios y en el juego; así como del desarrollo de capacidades físicas, estado funcional, $\mathrm{y}$ conocimientos teóricos, servirá para conocer la evolución del aprendizaje y asegurar su mejor dirección.

Algunas pautas metodológicas pueden contribuir a la ejecución efectiva de la evaluación como, por ejemplo: a) Reflejar el carácter diagnóstico, formativo, sumatorio y sistemático de la evaluación, b) Los ejercicios que constituyan tests pedagógicos, también se ejecutarán como contenido de las clases, c) Dentro de este proceso estará presente la autoevaluación del alumno y el colectivo, d) Utilizar el método de comparación relativa de la dinámica de los indicadores individuales y colectivos, e) Investigar la relación: contenido planificado y ejecutado - resultados de tests pedagógicos (incluyendo la evaluación funcional de la carga aplicada) - observaciones en los juegos, y f) Conocer el estado de salud y el rendimiento escolar del deportista.

En cuanto a la impartición de los contenidos antes mencionados en cada fase del proceso de enseñanzaaprendizaje, es preciso comprender que este no se produce de forma unidireccional, sino que de manera gradual se van incluyendo diversos contenidos, considerando los objetivos a alcanzar, las tareas a cumplir y la relación existente entre los contenidos; lo que 


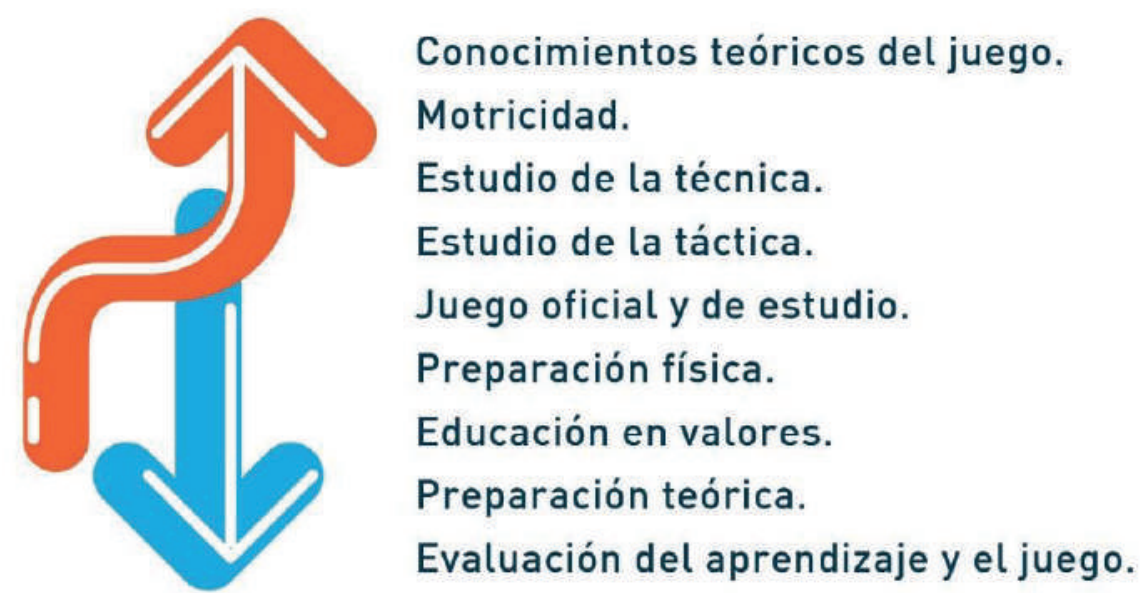

Figura 2. . Inclusión multidireccional de los contenidos en el proceso de enseñanza aprendizaje del juego.

Fuente: Elaboración propia.

determina la progresión en la enseñanza (ver Figura 2).

Estrategia didáctica del proceso de enseñanza aprendizaje del baloncesto en principiantes

Con el fin de alcanzar una adecuada aplicación de los fundamentos didácticos de la metodología y facilitar al profesor o entrenador deportivo un sistema de acciones y operaciones seleccionadas y organizadas en forma de tareas docentes, que aseguren la progresión, sistematicidad, asimilación y adaptabilidad de los contenidos en la marcha de la enseñanza; y considerando las ideas de González, Gutiérrez, Pastor y Fernández (2007), acerca de que la organización del proceso de enseñanza-aprendizaje debe llevarse a cabo estructurando etapas de desarrollo; se propone dividir el proceso en fases temporales.

Teniendo en cuenta, el potencial inicial de aprendizaje de los contenidos necesarios para jugar de los principiantes, el proceso de enseñanza se divide en dos fases: fase inicial de aprendizaje y fase de ampliación y consolidación de los contenidos del juego, cuyos objetivos, tareas y características se explican a continuación.

\section{Fase inicial de aprendizaje}

En la fase inicial, se crean las bases del ulterior aprendizaje del juego, en un tiempo que puede oscilar entre cuatro $\mathrm{y}$ seis meses, en dependencia de la frecuencia de clases o entrenamientos semanales. La orientación principal de la enseñanza en esta fase es: el dominio de los fundamentos técnicos, el inicio del aprendizaje de las acciones tácticas individuales y de grupo más elementales y sus principios, un acondicionamiento físico de base, el conocimiento de las reglas fundamentales del juego, la educación de valores; así como otros conocimientos teóricos que le permiten al principiante iniciarse en la práctica del deporte y desenvolverse de manera satisfactoria en un juego muy elemental, 
donde se aplican las reglas de forma no estricta, sino educativa.

\section{Tareas para la fase}

a) La primera tarea que tiene el profesor ante los alumnos es presentar el juego, después desarrollar un trabajo de motricidad general y relacionada al baloncesto.

b) Al mismo tiempo, comenzar el estudio de los elementos técnicos ofensivos por separado en condiciones sencillas, teniendo en cuenta el orden lógico establecido para cada grupo de elementos, los conocimientos precedentes necesarios y la aplicación de un conjunto de métodos y procedimientos para su enseñanza (Pardo, 2007).

c) Durante el estudio de los fundamentos técnicos deberán considerarse las tareas de la formación de la acción motora (Bogen, 1985).

d) En la medida que se logra el dominio elemental de los fundamentos técnicos por separado, se deben estudiar los fundamentos combinados.

e) De forma progresiva, con el dominio de los elementos técnicos combinados, iniciar el estudio de acciones tácticas individuales ofensivas más elementales, incluyendo conceptos del juego individual (Cemachko,1976).

f) Después de dominar los fundamentos técnicos ofensivos básicos para jugar de forma elemental, iniciar el estudio de las posturas y desplazamientos defensivos. g) Desde las primeras clases realizar juegos de estudio 5 vs. 5 y 3 vs. 3, ocupando aproximadamente el $20 \%$ del tiempo total de trabajo.

h) Desarrollar la preparación física multilateral, considerando la edad de los practicantes y los períodos sensibles de desarrollo de las capacidades físicas.

i) Conocer las reglas del minibaloncesto o del baloncesto de acuerdo a la categoría de los practicantes; así como reglas higiénicas de la actividad deportiva.

j) Desarrollar la atención de los niños en las clases, enseñar a contemplar de forma correcta el objeto de estudio, ejercitar la percepción del tempo de los ejercicios y de las acciones.

k) Educar valores ciudadanos, institucionales y relacionados con el contenido funcional del deporte que contribuyan a formar la personalidad del niño; así como controlar la marcha de su actividad en la escuela y las actividades fundamentales de su vida cotidiana.

Esta primera fase del proceso de enseñanza-aprendizaje se caracteriza por:

- La utilización en el trabajo técnico y táctico de los siguientes métodos: explicación y demostración, repetición, ejercicio competitivo, problemático, acciones simplificadas, juegos motores, pre-deportivos, y de estudio, acorde con el objetivo y contenido de la enseñanza; así como del nivel de conocimientos del practicante (Pardo,2007). 
- El ordenamiento y elección de las acciones técnicas y tácticas; así como los pasos para su enseñanza, se enfocan sin rigidez desde el aprendizaje.

- La enseñanza de los elementos técnicos con ambas manos (hábil y no hábil).

- La participación consciente y alegre de los jugadores en los ejercicios de la clase.

- La atención individual a los jugadores, no solo en el plano técnico, físico o táctico, sino también en otros aspectos de su vida.

- La correspondencia de los contenidos a impartir en esta etapa con el programa de enseñanza o plan de entrenamiento elaborado para el equipo, el nivel de preparación y la capacidad de aprendizaje de los jugadores, las orientaciones al respecto expuestas en el presente artículo y en la bibliografía recomendada, y las condiciones organizativas y materiales existentes.

- La aplicación en el desarrollo de la preparación física multilateral de: juegos motrices, relevos, gimnasia y atletismo, entre otros.

Es preciso aclarar que, en el desarrollo del programa de educación física de las escuelas relacionado con la enseñanza del baloncesto, esta fase inicial de aprendizaje puede tener una larga duración, no obstante; la participación en competencias escolares, y la práctica en horarios extras como trabajo individual del estudiante, acortarán su extensión.
Fase de ampliación y consolidación de los contenidos del juego

En esta fase, el baloncestista amplía su arsenal de elementos técnico - tácticos, los que aplica en el juego con regularidad $\mathrm{y}$ seguridad, domina acciones $\mathrm{y}$ principios del juego ofensivo y defensivo, continúa el desarrollo de sus capacidades físicas y es capaz de desempeñarse universalmente en un partido, sin demostrar un alto grado de especialización en una posición determinada, ni lograr altos rendimientos deportivos.

En la medida que avanza el proceso de enseñanza - aprendizaje del juego este se complejiza, aumentando las exigencias para los jugadores.

Las principales tareas que se deben acometer en esta fase, son las siguientes:

a) Dominar los fundamentos técnicos ofensivos y defensivos sigue siendo la tarea principal en las clases.

b) Cuando se comienzan a incluir ejercicios ofensivos con oposición defensiva, primero pasiva y después activa, se abordan las técnicas de oposición y apoderamiento defensivo, incluyéndose de forma progresiva las acciones tácticas individuales defensivas (Cemachko,1976).

c) Después de dominar las formas básicas de los fundamentos técnicos y sus combinaciones, se pasa a su consolidación y realización en condiciones cambiantes y de juego con oposición del adversario. 
d) Continuar el estudio de acciones tácticas individuales, incluyendo las referentes a posiciones o funciones en el equipo, las acciones de grupo ofensivas y defensivas según su ordenamiento lógico, de forma tal, que los jugadores aprendan sus principios.

e) Comenzar el estudio de las acciones de los jugadores en la lucha por el rebote ofensivo y defensivo.

f) Estudiar las acciones de equipo ofensivas y defensivas esenciales, precedidas de la debida preparación técnica para la táctica.

g) Iniciar el aprendizaje del juego u ofensiva de equipo por conceptos.

h) Participar con regularidad en juegos de estudio ( 5 vs. 5 y 3 vs. 3 ) y en juegos oficiales, incrementándose la cantidad de juegos con relación a la fase anterior.

i) Continuar el desarrollo de la preparación física multilateral de los jugadores, teniendo en cuenta sus edades.

j) Profundizar en el conocimiento de las reglas del juego, así como en otros aspectos referentes al entrenamiento deportivo, la cultura física y el deporte.

k) Continuar la educación de valores y actitudes relacionadas con el sentido del deber, dentro y fuera del equipo.

1) Mejorar las funciones psíquicas que propician la actividad motriz de los jugadores. Desarrollar rasgos volitivos y una adecuada disposición psíquica para las competencias y entrenamientos.

Esta segunda fase del proceso de enseñanza-aprendizaje se caracteriza por:

- La universalización en la formación técnico-táctica de los jugadores.

- De acuerdo con el nivel de conocimientos de los jugadores, pueden estudiarse algunos elementos técnicos y sus variantes que no son las fundamentales.

- El desarrollo del trabajo técnico con ambas manos (hábil y no hábil).

- La atención individual a los jugadores se hace más profunda; así como la motivación por la actividad que realiza.

- Los contenidos a impartir en esta etapa se establecerán, teniendo en cuenta los mismos elementos mencionados para la primera fase.

- La aplicación periódica durante el año de preparación de la planificación sobre la base de bloques de contenidos tácticos del programa de entrenamiento, sin dejar olvidar los contenidos aprendidos.

- En el trabajo técnico y táctico se continúa la utilización de los siguientes métodos: explicación y demostración, repetición, ejercicio competitivo, problemático, acciones simplificadas, juegos motores, pre-deportivos, y de estudio.

- Prestar atención a la reducción del 
tiempo de ejecución de los elementos técnicos.

- La utilización de métodos que permiten intensificar el entrenamiento como son: el método intervalo, circuito, complejo, influencia combinada, de tareas complejas de juego, y juego de estudio.

- La proporción de las clases dedicadas a la preparación física, técnica y táctica, con las que se destinan a los juegos de estudio y oficiales puede ser de $3: 1$.

- La realización de mini torneos de 3 $\mathrm{X} 3$ y 5 vs. 5, entre los integrantes del equipo y con invitados.

- La activa participación de los jugadores en los análisis del plan de preparación, los resultados de los tests pedagógicos y las competencias.

Relaciones entre los objetivos contenidos a impartir - estrategias de aprendizaje - métodos - medios procedimientos y evaluación de la enseñanza y del juego

Como se aprecia, los componentes del subsistema didáctico de la enseñanza del baloncesto a principiantes de cada fase son diferentes, dado que el proceso de enseñanza-aprendizaje para aquellos deportistas que no poseen conocimiento alguno o muy poco del baloncesto, se torna menos dinámico $\mathrm{y}$ denso, que el dirigido a aquellos que poseen los conocimientos básicos de este deporte. No obstante, en cada fase se establecen relaciones lógicas entre los objetivos contenidos a impartir - estrategias de aprendizaje - métodos - medios procedimientos y evaluación de la enseñanza y del juego, lo que determina la dinámica o progresión del proceso (ver Figura 3).

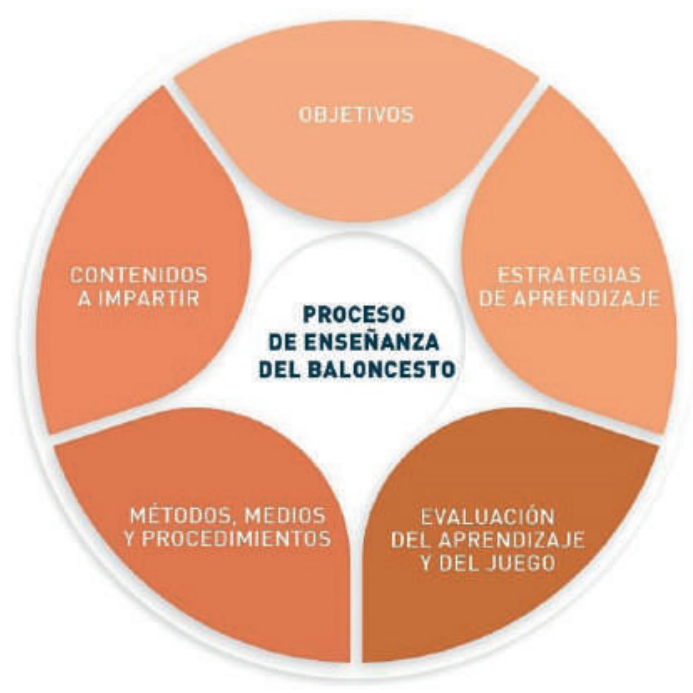

Figura 3. Relación entre los componentes del proceso de enseñanza del baloncesto.

Fuente: Elaboración propia.

Consideraciones sobre las particularidades de la enseñanza del minibaloncesto en el grupo o equipo de práctica

La mayoría de los equipos o grupos de práctica de principiantes en este deporte, se conforman por niños comprendidos en las edades de 9 a 12 años, los cuales competirán en el marco de las reglas del minibaloncesto, por lo que se hace necesario tener presente en el desarrollo del proceso de enseñanza - aprendizaje las características de dicha actividad.

El proceso de enseñanza aprendizaje del baloncesto adaptado a los niños, tiene como objetivo primordial que estos aprendan a jugar, disfruten de su práctica 
y reciban los beneficios que de esta emanan para su educación y desarrollo físico. Otra cosa importante es conseguir de que los chicos entiendan, que la suma de individualidades hace que el grupo funcione como un equipo y esto debe constituir un objetivo pedagógico de primer orden; puesto que es ahí donde, precisamente, radica la esencia del juego colectivo ser un equipo (Pidal, 2015). No debe desnaturalizarse el carácter competitivo del juego, elemento de gran valor educativo para el niño.

El modelo de metodología Padbal, representa un enfoque integral del proceso de enseñanza - aprendizaje del baloncesto dirigido a principiantes, que no se encuentra en la bibliografía especializada, aunque sí reflejado en los programas de preparación de baloncestistas en cuanto a contenido; pero no en cuanto a ordenamiento metodológico y fundamentación teórica del proceso de enseñanza. Este modelo de metodología se centra en la formación de la OFA en los jugadores, la cual determina en gran medida, el éxito en la ejecución de las acciones de juego.

Dando respuesta a la pregunta científica referente a ¿qué características presentan las metodologías para la enseñanza del baloncesto en los últimos tiempos? Y explicando las diferencias de la propuesta elaborada, puede afirmarse que esta difiere de los modelos utilizados en la enseñanza de los deportes de equipo (Gamero, 2015; Peráček y Peráčková, 2018). Empero, en relación al modelo estructural o tradicional, algunos aspectos son contemplados, como: iniciar el proceso de enseñanza por una de las habilidades específicas, y seguido a esto, integrar esta habilidad en situaciones simuladas de juego; mientras que se diferencia de dicho modelo, ya que parte del para qué y porqué de la acción al cómo. También, en el abordaje del estudio de la táctica de forma paralela con la técnica, aunque no esté formado el hábito motor en su totalidad, y en la libertad del profesor de elegir el método de enseñanza acorde con el contenido a impartir.

De igual modo, se presentan diferencias sustanciales con los modelos comprensivo, integrado y constructivista, ya que estos comienzan con la introducción de juegos modificados, con la intención de desarrollar un conocimiento táctico del juego y solo después se plantea la ejecución de la destreza, mientras que el modelo de metodología Padbal coloca al conocimiento técnico, como base del dominio táctico sin dejar de ser significativo el aprendizaje.

En el plano del aporte teórico de la investigación, los rasgos principales que distinguen el modelo de metodología Padbal de otros que se aplican en la actualidad en el baloncesto son: a) $\mathrm{Se}$ basa en fundamentos teóricos, metodológicos y prácticos de relevancia en la enseñanza de las acciones motrices y el juego; así como en la estructuración del proceso de entrenamiento. b) Abarca la enseñanza del baloncesto en su conjunto, no se tratan los elementos del proceso de manera aislada, como se presenta en otros trabajos sobre el tema. 
c) Considera las relaciones entre objetivo

- contenido - estrategia - evaluación como los factores que determinan la dinámica o progresión del proceso. d) Divide el proceso de enseñanza de este deporte para principiantes, en dos fases: fase inicial de aprendizaje y fase de ampliación y consolidación de los contenidos del juego, cuyos objetivos, tareas y características se explicaron con anterioridad, lo que permite la programación de este proceso, teniendo en cuenta las capacidades y conocimientos que va adquiriendo el jugador en el tiempo, aspecto que supera, en cuanto a dirección del proceso de enseñanza con principiantes, a otros trabajos que abordan esta temática. e) Orienta sobre qué bases organizar y tratar los contenidos de este deporte en las etapas antes mencionadas. f) Comprende la enseñanza de la táctica partiendo del conocimiento de sus principios, y enseñando a los jugadores a leer la situación de juego y reaccionar de manera adecuada. g) Orienta la aplicación de la evaluación funcional de la carga aplicada y la utilización del método de comparación relativa de la dinámica de los indicadores individuales $\mathrm{y}$ colectivos en la evaluación. h) Tiene presente en todo momento el papel protagónico del alumno en la construcción del proceso de enseñanza aprendizaje. i) Se adapta a las características de los contextos en que se desarrolla el proceso en cuestión.

El conjunto de orientaciones metodológicas, tareas y características de cada fase del proceso de enseñanza, que se plantean en el modelo de metodología
Padbal, poseen una significación práctica, ya que facilitan, especialmente a los jóvenes entrenadores y profesores de educación física, la labor de programar y organizar las clases, considerando la participación en competencias. $\mathrm{La}$ comprobación de la efectividad del modelo propuesto, en condiciones naturales de la enseñanza o el entrenamiento deportivo, permitirá elevar los conocimientos acerca del tema tratado, y responder con mayor profundidad a preguntas teóricas y prácticas que aún subsisten entre los especialistas.

\section{Conclusiones}

El análisis de la literatura científico metodológica sobre el problema de la presente investigación muestra que las metodologías existentes para la enseñanza del baloncesto a principiantes, exigen de perfeccionamiento, con el objetivo de elevar la efectividad y racionalidad de todo el proceso de enseñanza en cuestión. El modelo de metodología Padbal para la enseñanza del baloncesto, se sustenta en fundamentos didácticos no comprendidos totalmente en las metodologías que actualmente se utilizan en la práctica pedagógica, como son: los principios didácticos generales aplicados a la enseñanza de las acciones motoras, los principios generales y especiales de la preparación deportiva, la teoría sobre la dirección del aprendizaje de los conocimientos, la formación de las acciones y los conceptos, las tareas generales de la enseñanza del baloncesto, y la evaluación del proceso de enseñanza 
y del juego; teniendo como eje central la formación de la orientación fundamental de la acción en el jugador, lo cual supera, en cuanto a dirección del proceso de enseñanza con principiantes, a otros modelos de enseñanza que se utilizan en la práctica actualmente, además de facilitar la programación de la enseñanza de este deporte a los principiantes.

El establecimiento de dos fases para programar el proceso de enseñanza del juego en principiantes: la fase inicial de aprendizaje y la fase de ampliación y consolidación de los contenidos del juego, partiendo del potencial inicial de aprendizaje de los jugadores; así como de las orientaciones referentes a las tareas a cumplir en las mismas, sus contenidos y las características que en la práctica pedagógica reviste cada fase, constituye un aporte teórico de la investigación a la metodología de la enseñanza de este deporte y a su vez, una valiosa guía de trabajo para los profesores que, en los centros educacionales e instituciones deportivas, se encargan de la enseñanza del baloncesto.

El modelo de metodología propuesto está orientado a la formación a largo plazo de los baloncestistas en las etapas de iniciación del aprendizaje y comienzo de la estabilización de las acciones técnicas y tácticas del juego; las cuales se producen en las edades del minibaloncesto o las primeras categorías escolares, pero también puede ser aplicado en la educación física y la recreación con su debida adecuación a los objetivos, condiciones organizativas y materiales que se presenten en cada caso.

\section{Referencias}

Alonso, M. (2016). Planteamientos: De la técnica a la táctica o de la táctica a la técnica. Fbcv Blog.

Basketball for Coaches (2020). 73 Basketball drills and games for kids. (2020 Update). https://www.basketballforcoaches.com/b asketball-drills-and-games-for-kids/

Bogen, M. M. (1985). Fundamentos metodológicos de la teoría de la enseñanza de las acciones motrices. En Enseñanza de las acciones motrices. Mockva: Fizcultura Sport. B 4201000000-021/009[01]85/51-85

Carballo, M., y Guelmes, E. L. (2016). Algunas consideraciones acerca de las variables en las investigaciones que se desarrollan en educación. Revista Universidad y Sociedad, 8(1), 140-150.

Cárdenas, D., Alarcón, F., Piñar, M., Giménez, J., Padial, P., FericheCastanis, B., Campos, C., y Sixto, P. (2015). Enseñanza del Baloncesto, nivel 1. Federación Andaluza de Baloncesto.

Castillo, I. (2019). 5 Instrumentos de Investigación Instrumental. Lifeder.com

Cemachko, N. V. (1976). Tiexnika takticheskaia podgatovski basketbolistov. En Backetbol. Uchepnik dlia fiz. un-tob. Izd. 2-e perepab. B27. 7A8.1 Moskva: Fizcultura y Sport.

Cheng, F., y Sheng, Sh. (2018). A Study of College Basketball LAMS Teaching Model Centered on Learning Activities. International Journal of Emerging Technologies in Learning (iJET), 13(8), 210-224.

Erculj F., y Strumbelj, E. (2015). Basketball Shot Types and Shot Success in Different Levels of Competitive Basketball. Plos One, 10(6), 1-14. DOI: 10.1371/journal. pone. 0128885 . 
Famose, J. P., (2015). Aprendizaje motriz y resolución de problemas (y II). Revista de Educación Física, 32(2), 1-15.

Fessia, G. (2016). Enseñanza del Saber Táctico en Categorías Formativas de Básquetbol. [Tesis de Postgrado], Universidad Nacional de la Plata.

Gamero, M. (2015). Modelos de enseñanza-aprendizaje en los deportes de colaboración-oposición empleados por maestros en formación. [Tesis de Postgrado], Universidad de Extremadura.

Gayubo, A. (2017). La psicomotricidad, etapas y áreas. Propuesta aplicada al baloncesto. Fbcv Blog.

González E., Ibáñez S., y Feu, S. (2017). Diseño de Dos Programas de Enseñanza del Baloncesto Basados en Métodos de Enseñanza-Aprendizaje Diferentes. Revista de Ciencias del Deporte, 13(2), 131-152.

González, S., Gutiérrez D., Pastor, J., y Fernández, J. (2007). El proceso de enseñanzaaprendizaje del Baloncesto en las escuelas deportivas. Propuesta de programación para la categoría Benjamín. RETOS. Nuevas Tendencias en Educación Física, Deporte y Recreación, (11), 17-25.

Ibáñez, S., Feu, S., García-Rubio, J. (2019). Los procesos de formación y rendimiento en baloncesto. Progresos científicos para su mejora. Sevilla: Editorial Wanceulen.

Irina, O. V. (2015). Programa de estudio de baloncesto para la preparación inicial y grupos de entrenamiento. Red Social de Educadores ns.portal.ru

Mondoni, M. (2017). 27 Ejercicios para el desarrollo de los fundamentos del baloncesto. II Minibasket di Maurizio Mondoni.

Moti, G., y Wolde, S. (2018). Technical problems in teaching basketball practical session: The case of Grade 11 in Alamura Preparatory School. Journal of Physical Education and Sport Management, 9(4), 30-42.

Pardo, R. H. (2007). Particularidades del proceso de enseñanza del baloncesto. En Baloncesto para Niños y Jóvenes. Metodología para la enseñanza y el entrenamiento del juego. La Habana: Editorial Deportes.

Pavlovich, I. (2019). Siete principios de aprendizaje sistémico de calidad. Elitarium.ru.

Peráček, P., y Peráčková, J. (2018). Tactical Preparation in Sport Games and Motivational Teaching of Sport Games Tactics in Physical Education Lessons and Training Units. IntechOpenLimited. DOI: 10.5772/intechopen.75204

Pidal, J.C. (2015). Desde el Minibasket se enseña a hacer un equipo. Basketmania Blog.

Platonov, V. N. (2014). El sistema de entrenamiento de atletas en los deportes olímpicos. Teoría general y sus aplicaciones prácticas. Ucrania: Literatura olímpica.

Popclevich, A. V. (2019). Métodos de enseñanza para alumnos de 5-6 clases de juego de baloncesto en un colegio pequeño de pueblo.[Trabajo de grado], Universidad Pedagógica Humanitaria Estatal de Altansky.

Rebollo, J., Vizcaíno, C., Sáenz-López, P., y Fernández, E. (2015). Evaluation of an intervention program for the initiation to basketball aimed at teaching the game's rules. Revista de Psicología del Deporte, 24(3), 85-87.

Salazar, C. (2013). El conocimiento de los métodos de enseñanza del minibasquet y su repercusión en el aprendizaje, en las escuelas de las parroquias del gobierno autónomo del Cantón Rumiñahui de la 
Provincia de Pichincha. [Tesis de Grado], Universidad Técnica de Ambato.

Styopin V. S., Yelsukov A.N., y Goldberg F.I. (2020). Métodos del conocimiento científico. Portal Humanitario, https:/gtmarket.ru/concepts/6874.

Vladimirovich, V. (2015). Criterios de evaluación del control pedagógico en el Baloncesto Red Social de Educadores ns.portal.ru. 